\title{
Tunable Surface Wrinkling on Shape Memory Polymers with Application in Smart Micromirror
}

\author{
Yu Wang ${ }^{1, \dagger}$, Andres Villada ${ }^{1}$, Yao Zhai ${ }^{1}$, Zhanan Zou ${ }^{1}$, Yizhou Chen ${ }^{1}$, Xiaobo Yin $^{1,2}$, Jianliang \\ $\mathrm{Xiao}^{1, *}$ \\ ${ }^{1}$ Department of Mechanical Engineering, University of Colorado Boulder, Boulder, Colorado \\ 80309, USA \\ ${ }^{2}$ Material Science and Engineering Program, University of Colorado Boulder, Boulder, Colorado \\ 80309, USA \\ *Email: jianliang.xiao@colorado.edu
}

\begin{abstract}
Surfaces with tunable topological features enable important applications, such as optical devices, precision metrology, adhesion, and wetting. In this study, we demonstrate a facile method to fabricate and control the surface morphologies by combining thin film wrinkling and thermal expansion. This approach utilizes self-assembled surface wrinkling induced by shape recovery of shape memory polymers and localized thermal expansion caused by Joule heating. Recovering the prestrain in the SMP substrate induces global wrinkling of the thin film on the substrate. Joule heating in the SMP by a heating wire embedded in the substrate induces thermal expansion of the substrate in a localized area, which leads to disappearance of the wrinkling pattern. This effect is reversed when heating is stopped, leading to reversible and repeatable tuning of the surface morphology in a controllable localized surface region. With metal coating, the SMP surface can be switched from specular to diffuse reflectance with respond to external Joule heating. Finally, we demonstrate a smart micromirror device with its diffuse reflectance tunable between $13.5 \%$ to $81.9 \%$ in visible light region. This approach provides a method to modulate surface diffusivity by controlling its surface morphologies, with potential applications in optical display and optical microelectromechanical (MEMS) devices.
\end{abstract}




\section{Introduction}

Topological features on the surface were shown to have many important applications, such as optical devices, ${ }^{1-3}$ precision metrology, ${ }^{4,5}$ smart adhesion ${ }^{6-9}$ and wetting. ${ }^{10,11}$ Thin film wrinkling on soft substrate is a simple and low-cost method to fabricate controllable patterns on the surface either in big areas or along designed paths. ${ }^{12-15}$ A lot of efforts have been invested to investigate different means of realizing thin film wrinkling on soft substrates, including surface oxidation of polydimethylsiloxane (PDMS), ${ }^{12,16}$ transfer printing of thin films onto prestretched substrates, ${ }^{17,18}$ swelling, ${ }^{19}$ and laser writing. ${ }^{14,15}$ Wrinkling of many different thin film materials were also realized, such as metals, ${ }^{12,20}$ semiconductors, ${ }^{17,21}$ nanomaterials, ${ }^{22-25}$ and polymers. ${ }^{8}$

Shape memory polymers (SMPs) are a type of smart materials that respond to environmental stimuli and could find interesting applications in many areas. ${ }^{26-28} \mathrm{~A}$ few studies have explored thin film wrinkling on SMPs, ${ }^{29-34}$ including controlled patterning, ${ }^{32}$ mechanics of wrinkling and evolution, ${ }^{34}$ and localized shape recovery induced localized wrinkling. ${ }^{33}$

In this study, we demonstrate a simple method to fabricate wrinkling pattern on SMP and to tune the wrinkling pattern in a controllable fashion by joule heating induced thermal expansion. By accurately control the joule heating induced thermal expansion, the wrinkling pattern can be turned into flat surface in a localized area, which was used to fabricate a tunable micromirror with drastic change in optical reflectivity.

\section{Methods and materials}

SMP synthesis SMP was synthesized by mixing the monomer tert-butyl acrylate (tBA) and crosslinker poly (ethylene glycol) dimethacrylate (PEGDMA) at ratio 4:1, and 2, 2-dimethoxy-2phenylacetophenone was added as the photoinitiator. ${ }^{35,36}$ The solution was then injected into a mold made by glass slides, with a piece of heating wire (FeCrAl Alloy, Diameter $127 \mu \mathrm{m}$, 
Resistance 32.1 to 37.7 ohms/foot) placed at the center of the mold. After being cured under UV lamp for 5 minutes, the SMP was then post-cured in the oven at $80^{\circ} \mathrm{C}$ for 1 hour. ${ }^{35,36}$ This formula of SMP gives the glass transition temperature $\left(T_{g}\right)$ to be $\sim 42{ }^{\circ} \mathrm{C} .{ }^{37}$

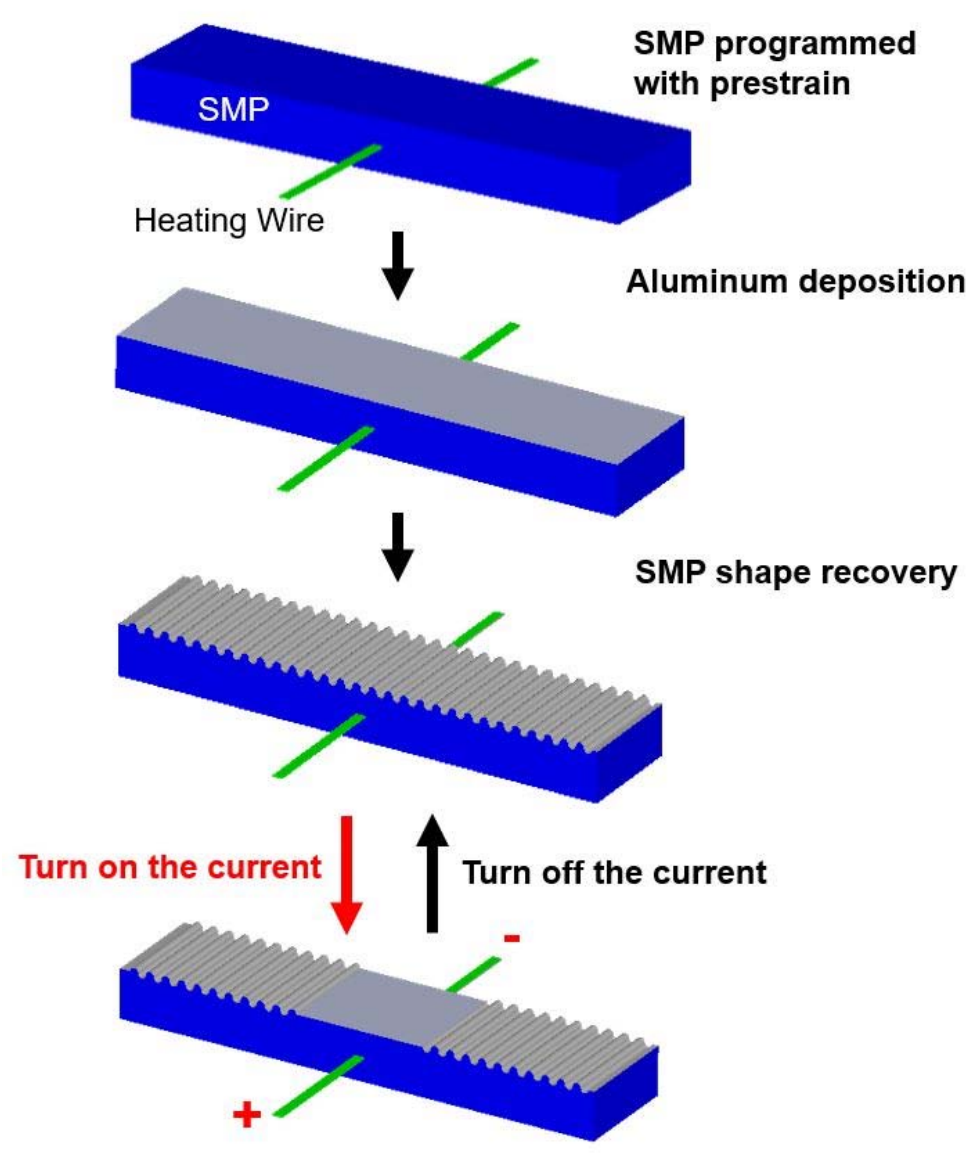

Figure 1. Schematic illustration of fabrication process to realize wrinkling on SMP and to locally flatten the surface by using joule heating.

Wrinkling pattern fabrication and tuning As shown in Fig. 1, the SMP substrate was uniaxially prestreched with $5 \%$ strain at $60{ }^{\circ} \mathrm{C}$, using Dynamic Mechanical Analysis (DMA) tester (TA Instruments, DMA Q800). After the sample was quenched to $25^{\circ} \mathrm{C}$, the strain was released, and the temporary and programmed shape was kept. Then, a layer of aluminum thin film of thickness $100 \mathrm{~nm}$ was deposited on the surface of programmed SMP substrate using a thermal evaporator. Afterwards, the SMP was heated to $60{ }^{\circ} \mathrm{C}$ to induce shape recovery in the substrate, leading to 
wrinkling of the aluminum thin film on the SMP. To achieve a crack free thin film surface and significant tunability of wrinkling morphology, $5 \%$ prestrain was chosen for this study.

To tune the wrinkling pattern, the heating wire was connected to a DC power supply. After the current was turned on, the SMP was heated with desired power and duration. Thermal expansion of the SMP due to joule heating led to the disappearance of the pattern. The wrinkling pattern forms again when the power was turned off and SMP cooled down. In this way, the surface morphology can be effectively tuned.

\section{Results and discussions}

Figures 2a-h show the optical microscope images of the surface morphology during reversible tuning process. The surface was flat after thin film deposition (Fig. 2a), and wrinkling formed after the SMP substrate was recovered (Fig. 2b). After recovering, the wrinkle profile showed sinusoidal shape with wavelength $7.1 \mu \mathrm{m}$ and amplitude $255 \mathrm{~nm}$ (Fig. 2i). To tune the wrinkling pattern, heating wire embedded in the SMP substrate was turned on with current $\mathrm{I}=0.45$ A. Due to thermal expansion, the wavy morphology gradually diminished, and after 3 minutes, the surface became almost flat, with amplitude down to $25 \mathrm{~nm}$ (Fig. 2c and Fig. 2i), comparable to the surface roughness of aluminum thin film on SMP substrate. ${ }^{38}$ This wrinkling-flat process is repeatable. To form the wrinkling pattern again, the heating wire was turned off and the SMP was cooled down, the wrinkling pattern reached its initial state after 5 minutes (Figs. $2 \mathrm{~d}$ and 2i). The profile showed good agreement with that of Fig. $2 \mathrm{~b}$ when SMP was initially recovered to form wrinkling. This tuning process was repeated for two more cycles, as shown in Figs. 2e-2h. As shown in Fig. 2i, the profiles of the flat and wrinkles states match well with their correspondents in different cycles, demonstrating excellent repeatability and reversibility. 

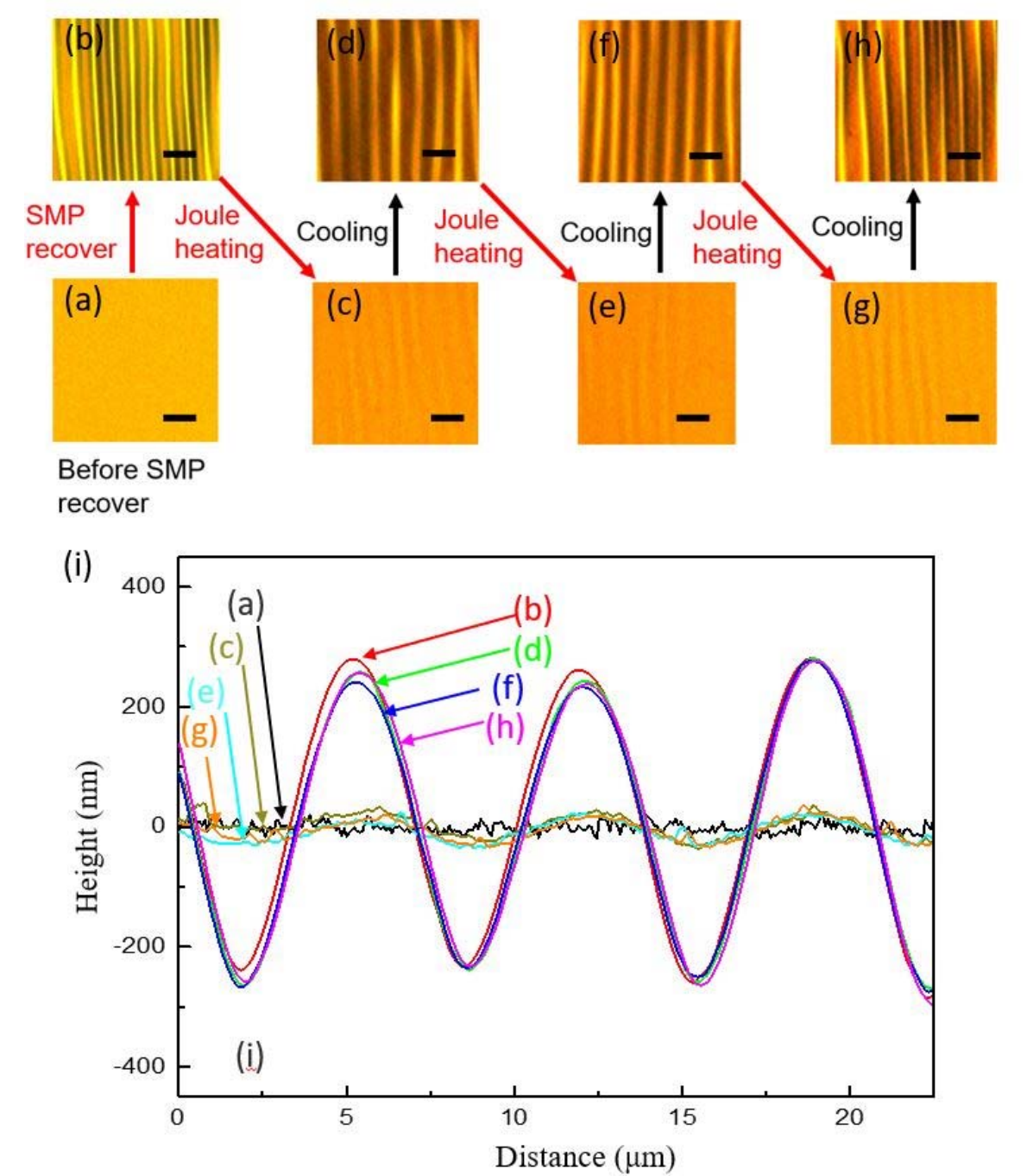

Figure 2. Optical microscope images of the surface in the reversible and repeatable tuning process ( $\mathrm{a}-\mathrm{h}$ ), and the corresponding profiles (i), for Joule heating with current $\mathrm{I}=0.45 \mathrm{~A}$ and heating time for 3 minutes, and cooling for 5 minutes. (Scale bar $5 \mu \mathrm{m}$ ).

The dependence of wrinkle wavelength and amplitude over distance from the heating wire are plotted in Figs. 3a and 3b, respectively. The inserts mark the locations of the measurements, which are $0 \mathrm{~mm}, 1 \mathrm{~mm}, 2 \mathrm{~mm}, 3 \mathrm{~mm}$, and $4 \mathrm{~mm}$ from the heating wire. Before heating, the wrinkling profile was induced by recovery of the SMP substrate. The strain recovery was uniform along the sample and led to a uniform distribution of the wrinkle wavelength and amplitude (red dots in Figs. $3 \mathrm{a}$ and 3b). After Joule heating for 3 minutes, the wrinkle wavelength remained 
unchanged (blue dots in Fig. 3a), but the wrinkle amplitude showed symmetrical distribution, which decreases towards the heating wire (blue dots in Fig. 3b). The amplitude in the center (above the heating wire) was the smallest $(25 \mathrm{~nm})$, and it increased to $61 \mathrm{~nm}$ at $1 \mathrm{~mm}, 83 \mathrm{~nm}$ at $2 \mathrm{~mm}$, $175 \mathrm{~nm}$ at $3 \mathrm{~mm}$, and $242 \mathrm{~nm}$ at $4 \mathrm{~mm}$. At $4 \mathrm{~mm}$, it was comparable to that before Joule heating. Such phenomenon was due to localized heating induced nonuniform but symmetrical thermal expansion in SMP. When the power to the heating wire was turned on, the temperature decreased with the distance from heating source. The temperature in the center was the highest and decreased gradually and symmetrically along both sides, which led to decreasing thermal expansion of the SMP with increasing distance from the heating wire. According to the established thin film buckling theory ${ }^{17,39}$, the wavelength of a buckled thin film subject to a tensile strain doesn't change with the applied strain, while the amplitude decreases with increasing tensile strain, which follows the equation given below

$$
A=h \sqrt{\frac{\varepsilon_{p r e}-\varepsilon_{a}}{\varepsilon_{c}}-1},
$$

where $h$ is the film thickness, $\varepsilon_{\text {pre }}$ is the recovered SMP program strain, $\varepsilon_{a}$ is the applied strain due to thermal expansion, and $\varepsilon_{c}$ is the critical buckling strain.

A Finite Element Analysis (FEA) was also conducted to study the variation of wrinkle wavelength and amplitude over distance in a thermal cycle. To capture the thermal-mechanical behavior of SMP, a thermal-mechanical constitutive model was implemented in the FEA software package ABAQUS (Simulia, Providence, RI) as a user material subroutine (UMAT). This model was developed by Westbrook et al., and it was shown to be able to accurately predict the thermalmechanical response of SMPs. ${ }^{37}$ In the simulation, the SMP substrate was modeled using quadratic coupled temperature-displacement hybrid elements (CPE8HT), and the aluminum thin film was modeled using quadratic coupled temperature-displacement elements (CPE8T). The 
same process as in experiment described previously was adopted to generate thin film wrinkling on SMP. To induce localized heating and flattening of the thin film, a concentrated heat flux was applied in an embedded element, and the heat flux was calculated according to the electric current and resistance of the heating wire used in the experiment. The FEA results are plotted in Figs. 3a and $3 \mathrm{~b}$, and they show good agreement with experiments for both before and after Joule heating. Figure 3(c) shows the dependence of wrinkle amplitude on temperature in the tuning process, obtained by FEA simulation. The wrinkle amplitude decreases with the increase of temperature, which is due to thermal expansion of the SMP. With change of the temperature, this bilayer system achieved 10 times tunability of the wrinkle amplitude. With understanding of the phenomenon and mechanism, this method is possible to be extended to other stiff thin films, including metals, oxides and ceramics, for different applications in various areas.
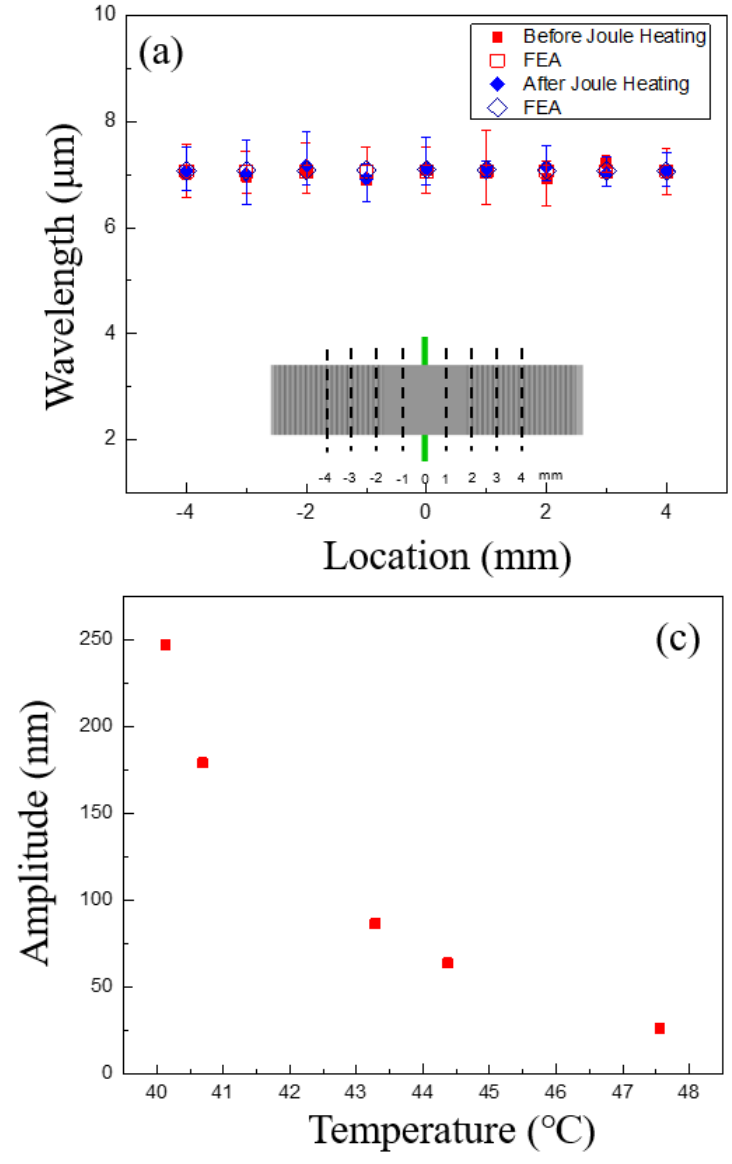

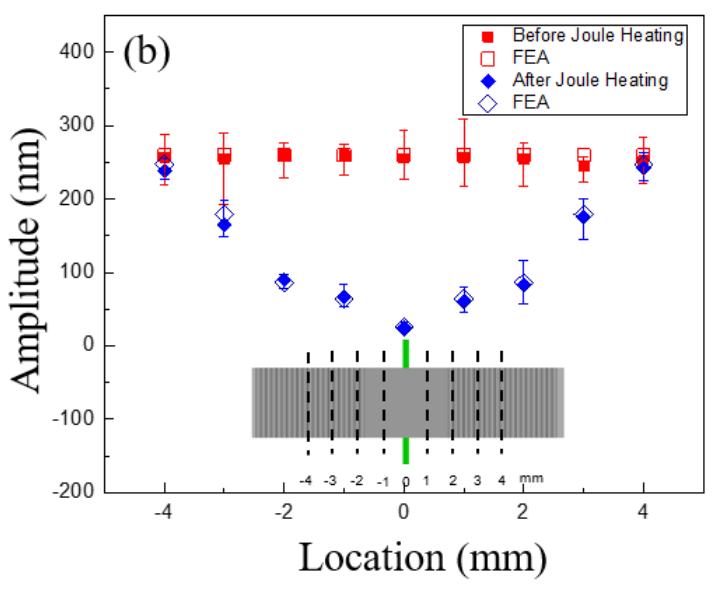

Figure 3. (a) Wavelength and (b) amplitude distribution before and after Joule heating with current $\mathrm{I}=0.45 \mathrm{~A}$ and heating time for 3 minutes, obtained from experiments and FEA simulations, (c) dependence of amplitude on temperature in the tuning process. 
By controlling the heating power and time, the size of the heating region can be controlled, and in return the size of the region of tunable surface morphology can be controlled. Figure 4 exhibits the size of the region of tunable surface morphology by using Joule heating versus the heating current. Here the boundaries of the region of tunable surface morphology are determined by the profile of wrinkling. The boundary between the wrinkling area and flat area is defined when the amplitude of wrinkling decreases to $50 \mathrm{~nm} .{ }^{33}$ The size of the tunable region increases from 2.2 $\mathrm{mm}$ with $0.45 \mathrm{~A}$ heating current to $6.7 \mathrm{~mm}$ with $0.8 \mathrm{~A}$ heating current. The responding speed of surface morphology tuning strongly depends on the heating power. Figure $4 \mathrm{~b}$ shows the dependence of the responding time on the electric current. The time shown in Fig. $4 \mathrm{~b}$ is how long it takes to reach an almost flat surface. The responding time decreases significantly with the increase of the electric current, from $160 \mathrm{~s}$ at $0.45 \mathrm{~A}$ to $20 \mathrm{~s}$ at $0.8 \mathrm{~A}$.
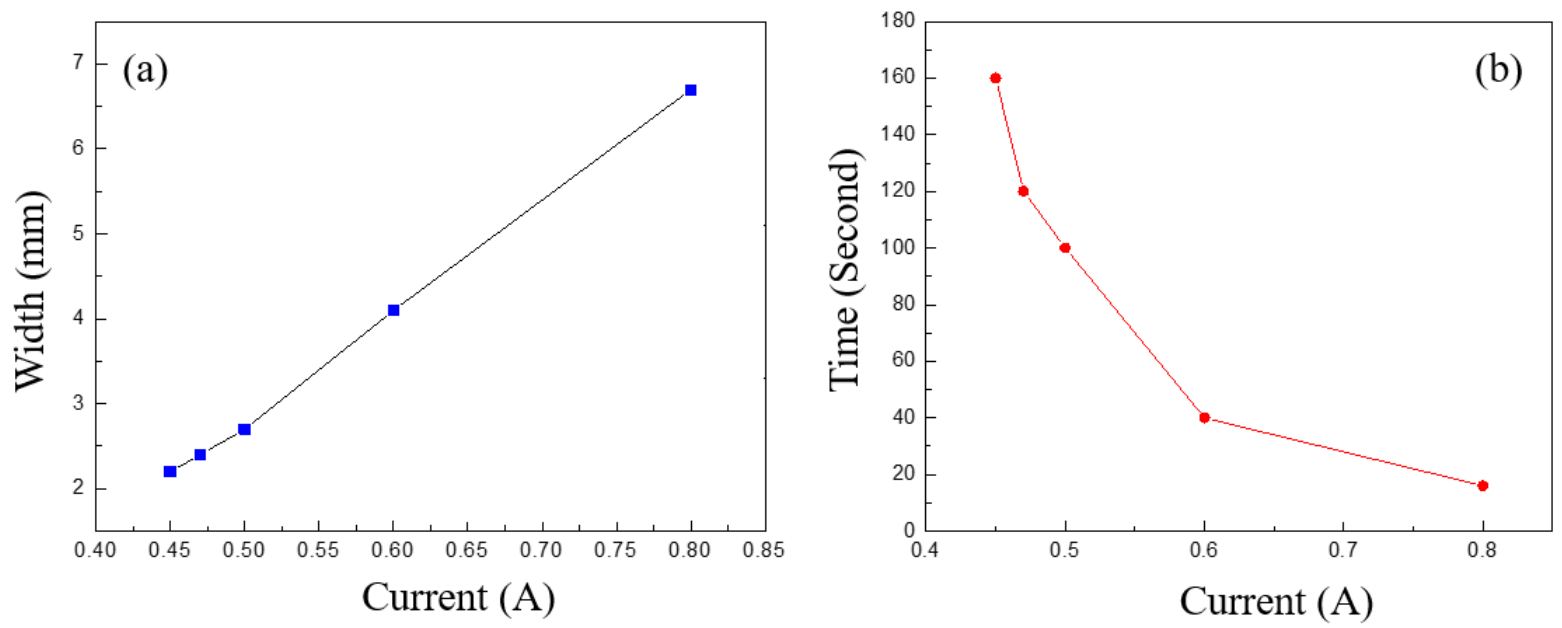

Figure 4. (a) The size of the heating region can be tuned by controlling the heating current, (b) Dependence of responding time on electric current

Micromirrors are important components in various highly miniaturized optical devices, such as laser projection displays, optical microelectromechanical (MEMS) devices, and portable 
scanning grating spectrometers. ${ }^{40}$ Each mirror has ON and OFF states that determine the direction of reflected light. Here a smart micromirror device with similar function is demonstrated by tuning the surface morphology to change the diffuse reflectance, as shown in Fig. 5. The inserted figure shows the wrinkle (rough) and flat (mirrored) surfaces before and after Joule heating, respectively. The diffuse reflection spectra were measured following standard test measurement ASTM STP478. ${ }^{41}$ The integrating sphere was applied to collect diffusive reflection light reflected by SMP surface at wrinkled and flat state. After $100 \mathrm{~nm} \mathrm{Al} \mathrm{deposition,} \mathrm{the} \mathrm{surface} \mathrm{was} \mathrm{flat} \mathrm{with}$ diffuse reflectance $13.5 \%$ (light wavelength $400 \mathrm{~nm}$ ). After the recovery of the SMP substrate, wrinkling formed and led to the increase of the diffuse reflectance to $81.9 \%$ (light wavelength 400 $\mathrm{nm})$. It is due to the dramatically improved light scattering effect introduced by the thin film wrinkling. ${ }^{42}$ When electric current was turned on, Joule heating flattened the surface and the diffuse reflectance decreased to $14.5 \%$, which is very close to that of the originally flat surface. After cooling down, wrinkling formed again, and the diffuse reflectance increased to $82.6 \%$. Switch of the diffuse reflectance is repeatable, and testing results of two more cycles are included in Fig. 5. Significant difference of the diffuse reflectance before and after Joule heating allows the device to switch between ON and OFF states. In this paper, the flat and wrinkled surfaces are defined as ON and OFF states, which has significant difference of diffuse reflectance. In the real application, lenient standard, for example, smaller difference of the diffuse reflectance can be considered as the state changes to define the ON/OFF states. Higher heating power and lenient standard of ON/OFF states can decrease the responding time significantly. This study provides a new approach to fabricate and tune the micromirror with much lower cost. 


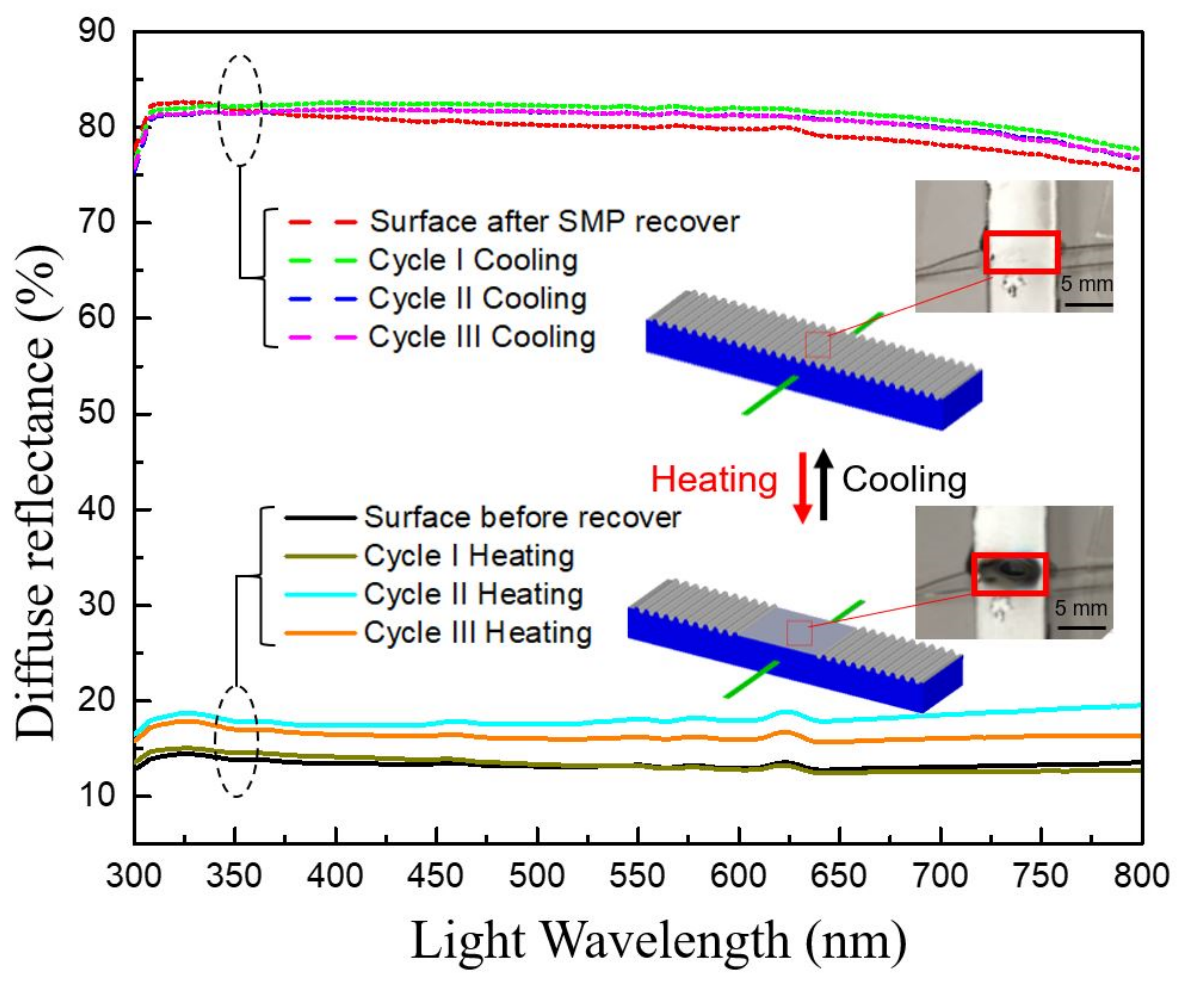

Figure 5. Tunable diffuse reflectance spectra of the surface by Joule heating.

For the tunable micromirror, coefficient of thermal expansion (CTE) of the substrate is important. If the CTE is too small, it requires very high temperature to flatten the surface by thermal expansion, which increases power consumption and could also limit the application in temperature sensitive systems. On the other hand, too big CTE makes the device hard to control, as thermal expansion induced tension strain could damage the thin film. ${ }^{31}$ For the SMP substrate used here, the rubbery state $\mathrm{CTE}$ is $2.35 \times 10^{-4} /{ }^{\circ} \mathrm{C} .{ }^{37}$ Joule heating by heating wire produces thermal expansion that counteracts the pretrain and flattens the surface for low diffusion reflectance. Therefore, thermal properties of the substrate play important roles. With increasing thermal expansion coefficient, the temperature required to achieve the same level of tunable surface morphology decreases. Thermal conductivity determines the speed of conducting heat. 
Higher thermal conductivity leads to higher response speed in the tuning process. Thermal capacity determines the amount of thermal energy required for a desired temperature increase. With fixed electric power, lower thermal capacity results in lower thermal energy consumption and higher tuning speed.

The wrinkle amplitude plays a key role in optical properties of the surface wrinkling pattern. Figure 6 plots diffuse reflectance of the surface wrinkling pattern with different amplitudes for light wavelength $300 \mathrm{~nm}, 500 \mathrm{~nm}$, and $700 \mathrm{~nm} .0$ amplitude corresponds to the flat surface of the as-deposited Al film on SMP. With the increase of the amplitude, the diffuse reflectance increases due to light scattering caused by the rough thin film surface pattern. ${ }^{42}$ Tuning the wrinkle amplitude can be an effective approach to achieve desired diffuse reflectance.

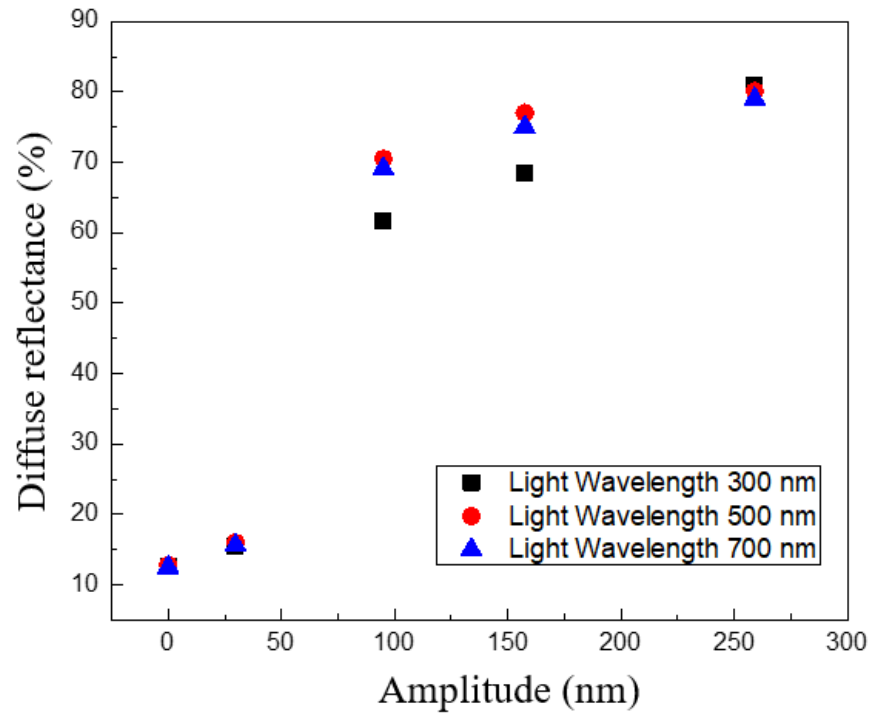

Figure 6. Dependence of diffuse reflectance on amplitude.

\section{Conclusions}

To conclude, a simple and low-cost method to form and tune surface morphologies is presented. This method utilizes thin film-SMP substrate bilayer system and a heating wire embedded in the substrate. Recovering prestreched SMP substrate induces wrinkling of the film 
and Joule heating by heating wire flattens the wrinkling in a controllable localized region due to thermal expansion. The wrinkling reforms after the Joule heating stops and the pattern recovers to its initial geometry. And this reversible tuning of surface morphology is repeatable. Such tunable surface wrinkling pattern is utilized to realize a smart micromirror device with ON and OFF states. This approach can be easily extended to other stiff thin films, including metals, oxides and ceramics, for potential applications in various areas, such as optics, surface adhesion, and wetting.

\section{Acknowledgment}

The financial support from NSF (CMMI-1405355) is gratefully acknowledged. We thank the seed grant support from the Soft Materials Research Center under NSF MRSEC Grant No. DMR-1420736. We also thank Prof. Jerry Qi at Georgia Tech for sharing the UMAT of SMP for finite element simulation.

\section{Conflicts of interest}

There are no conflicts of interest to declare.

\section{Present Address}

$\dagger$ Department of Nanomedicine, Houston Methodist Research Institute, Houston, Texas 77030, United States

\section{References}

1 C. Yu, K. O’Brien, Y. Zhang, H. Yu, and H. Jiang, Applied Physics Letters 96, 04111 (2010).

2 D. Ge, E. Lee, L. Yang, Y. Cho, M. Li, D. S. Gianola, and S. Yang, Advanced materials 27, 2489 (2015).

3 Z. Li, Y. Zhai, Y. Wang, G. M. Wendland, X. Yin, and J. Xiao, Advanced Optical Materials 5 (2017).

4 T. Ma, H. Liang, G. Chen, B. Poon, H. Jiang, and H. Yu, Optics express 21, 11994 (2013).

5 C. M. Stafford, C. Harrison, K. L. Beers, A. Karim, E. J. Amis, M. R. VanLandingham, H. C. Kim, W. Volksen, R. D. Miller, and E. E. Simonyi, Nature materials 3, 545 (2004). 6 E. P. Chan, E. J. Smith, R. C. Hayward, and A. J. Crosby, Advanced materials 20, 711 (2008). 
P. Lin, S. Vajpayee, A. Jagota, C. Hui, and S. Yang, Soft Matter 4, 1830 (2008).

Y. Wang and J. Xiao, Soft Matter 13, 5317 (2017).

T. Xie and X. Xiao, Chemistry of Materials 20, 2866 (2008).

Jun Young Chung, Jeffrey P. Youngblood, and Christopher M. Stafford, Soft Matter 3, 1163 (2007).

S. G. Lee, D. Y. Lee, H. S. Lim, D. H. Lee, S. Lee, and K. Cho, Advanced materials 22, 5013 (2010).

N. Bowden, S. Brittain, A. G. Evans, J. W. Hutchinson, and G. M. Whitesides, Nature 393, 146 (1998).

N. Bowden, W. T. S. Huck, K. E. Paul, and G. M. Whitesides, Applied Physics Letters 75, 2557 (1999).

M. W. Moon, S. H. Lee, J. Y. Sun, K. H. Oh, A. Vaziri, and J. W. Hutchinson,

Proceedings of the National Academy of Sciences of the United States of America 104, 1130 (2007).

C. F. Guo, V. Nayyar, Z. Zhang, Y. Chen, J. Miao, R. Huang, and Q. Liu, Advanced materials 24, 3010 (2012).

Wilhelm TS Huck, Ned Bowden, Patrick Onck, Thomas Pardoen, John W Hutchinson, and George M Whitesides, Langmuir : the ACS journal of surfaces and colloids 16, 3497 (2000).

D. Khang, H. Jiang, Y. Huang, and J. A. Rogers, Science 311, 208 (2006).

Hanqing Jiang, Dahl-Young Khang, Huiyang Fei, Hoonsik Kim, Yonggang Huang,

Jianliang Xiao, and John A. Rogers, Journal of the Mechanics and Physics of Solids 56, 2585 (2008).

H. S. Kim and A. J. Crosby, Advanced materials 23, 4188 (2011).

Pei-Chun Lin and Shu Yang, Applied Physics Letters 90, 241903 (2007).

A. J. Baca, J. H. Ahn, Y. Sun, M. A. Meitl, E. Menard, H. S. Kim, W. M. Choi, D. H.

Kim, Y. Huang, and J. A. Rogers, Angewandte Chemie 47, 5524 (2008).

J. Xiao, H. Jiang, D. -Y Khang, J. Wu, Y. Huang, and J. A. Rogers, Journal of Applied Physics 104, 033543 (2008).

Dahl-Young Khang, Jianliang Xiao, Coskun Kocabas, Scott MacLaren, Tony Banks, Hanqing Jiang, Yonggang Y Huang, and John A Rogers, Nano letters 8, 124 (2008). Y. Wang, J. Song, and J. Xiao, Journal of Physics D: Applied Physics 46, 125309 (2013).

Z. Wang, D. Tonderys, S. E. Leggett, E. K. Williams, M. T. Kiani, R. S. Steinberg, Y.

Qiu, I. Y. Wong, and R. H. Hurt, Carbon N Y 97, 14 (2016).

L. Sun, W. M. Huang, Z. Ding, Y. Zhao, C. C. Wang, H. Purnawali, and C. Tang, Materials \& Design 33, 577 (2012).

WM Huang, Z Ding, CC Wang, J Wei, Y Zhao, and H Purnawali, Materials Today 13, 54 (2010).

Andreas Lendlein, Hongyan Jiang, Oliver Jünger, and Robert Langer, Nature 434, 879 (2005).

C. Fu, A. Grimes, M. Long, C. G. L. Ferri, B. D. Rich, S. Ghosh, S. Ghosh, L. P. Lee, A. Gopinathan, and M. Khine, Advanced materials 21, 4472 (2009).

Y. Zhao, W. M. Huang, and Y. Q. Fu, Journal of Micromechanics and Microengineering 21, 067007 (2011). 
J. Li, Y. An, R. Huang, H. Jiang, and T. Xie, ACS applied materials \& interfaces 4, 598 (2012).

Xueju Wang, Xiaogang Guo, Jilong Ye, Ning Zheng, Punit Kohli, Dongwhi Choi, Yi Zhang, Zhaoqian Xie, Qihui Zhang, and Haiwen Luan, Advanced materials 31, 1805615 (2019).

Yu Wang, Yao Zhai, Andres Villada, Sabrina N David, Xiaobo Yin, and Jianliang Xiao, Applied Physics Letters 112, 251603 (2018).

Y. Wang, K. Yu, H. J. Qi, and J. Xiao, Soft Matter 13, 7625 (2017).

C. M. Yakacki, R. Shandas, C. Lanning, B. Rech, A. Eckstein, and K. Gall, Biomaterials 28, 2255 (2007).

Qi Ge, Xiaofan Luo, Erika D. Rodriguez, Xiao Zhang, Patrick T. Mather, Martin L. Dunn, and H. Jerry Qi, Journal of the Mechanics and Physics of Solids 60, 67 (2012). Kristofer K. Westbrook, Philip H. Kao, Francisco Castro, Yifu Ding, and H. Jerry Qi, Mechanics of Materials 43, 853 (2011).

Yu Wang, Qingyang Sun, and Jianliang Xiao, Applied Physics Letters 112, 081602 (2018).

Y. Wang, Z. Li, and J. Xiao, Journal of Electronic Packaging 138, 020801 (2016). Pamela Rae Patterson, Dooyoung Hah, Makoto Fujino, Wibool Piyawattanametha, and Ming C Wu, Optomechatronic Micro/Nano Components, Devices, and Systems 5604, 195 (2004).

E Barkman, in Appearance of Metallic Surfaces (ASTM International, 1970).

Haimin Zhang, Yanhe Han, Xiaolu Liu, Porun Liu, Hua Yu, Shanqing Zhang, Xiangdong Yao, and Huijun Zhao, Chemical Communications 46, 8395 (2010).

\section{Figure captions}

Figure 1. Schematic illustration of fabrication process to realize wrinkling on SMP and to locally flatten the surface by using joule heating.

Figure 2. Optical microscope images of the surface in the reversible and repeatable tuning process (a-h), and the corresponding profiles (i), for Joule heating with current $\mathrm{I}=0.45 \mathrm{~A}$ and heating time for 3 minutes, and cooling for 5 minutes. (Scale bar $5 \mu \mathrm{m}$ ).

Figure 3. (a) Wavelength and (b) amplitude distribution before and after Joule heating with current $\mathrm{I}=0.45 \mathrm{~A}$ and heating time for 3 minutes, obtained from experiments and FEA simulations, (c) dependence of amplitude on temperature in the tuning process.

Figure 4. (a) The size of the heating region can be tuned by controlling the heating current, (b) dependence of responding time on electric current. 
Figure 5. Tunable diffuse reflectance spectra of the surface by Joule heating. Figure 6. Dependence of diffuse reflectance on amplitude. 